\title{
Sittlichkeitsprozesse gegen katholische Ordensleute und Priester in der NS-Zeit
}

\author{
Eine Relektüre nach 5 o Jahren
}

\author{
Hans Günter Hockerts
}

Joseph Goebbels zog alle Register der Demagogie, als er am 28. Mai 1937 in der Berliner Deutschlandhalle eine antiklerikale Massenkundgebung veranstaltete. „Herdenmäßige Unzucht“ habe sich in der katholischen Kirche ausgebreitet - das war sein Leitmotiv. „Tausende von kirchlichen Sexualverbrechern“ seien auf „planmäßige sittliche Vernichtung Tausender von Kindern und Kranken“ aus. Den bischöflichen Aufsichtsbehörden warf er „himmelschreiende Verantwortungslosigkeit“ vor; dem Klerus attestierte er einen „allgemeinen Sittenverfall, wie er in diesem erschreckenden und empörenden Ausmaß kaum noch einmal in der gesamten Kulturgeschichte der Menschheit festzustellen“ sei. Der Propagandist verstand es, sein Publikum - überwiegend Berliner SA-Formationen - mit der eingehenden Schilderung von „Scheußlichkeiten“ regelrecht in Rage zu bringen. Orkane des Beifalls brachen aus, wenn er Drohungen ausstieß. Zurufe wie „Aufhängen!“ waren zu hören.

Auch im Radio. Denn die Rede wurde von allen deutschen Rundfunkstationen übertragen, und tags darauf mussten sämtliche deutschen Zeitungen Teilabdrucke bringen. So kam diese Hetztirade in jede Stadt und jedes Dorf und beinahe in jedes Haus. Sie bildete den Gipfel einer Kampagne, die 1936/37 in den gelenkten deutschen Medien viel Raum einnahm und phasenweise so radikal verlief, dass der Münsteraner Bischof Galen von einem „Giftgasangriff“1 sprach. Der mediale Feldzug basierte angeblich auf über 1 ooo aktuellen Strafverfahren gegen Ordensangehörige und Priester, die zeitgenössisch als Sittlichkeitsprozesse bezeichnet wurden.

In meiner anno 1971 publizierten zeithistorischen Dissertation habe ich diese Kampagne untersucht und die leitenden Fragen auf die nationalsozialistische Herrschaftstechnik, die Rolle der Justiz und die Reaktion der Kirche gerichtet. Den übergreifenden Interpretationsrahmen bildete der Konflikt zwischen

1 Niederschrift über die Plenarkonferenz des deutschen Episkopats, 24.-26.8.1937, in: Ludwig Volk (Hrsg.), Akten deutscher Bischöfe über die Lage der Kirche 1933-1945, Bd. IV: 1936-1939. Mainz 1981, 329-338, Zitat 331.

(C) HANS GÜNTER HOCKERTS, 2022 | DOI:10.30965/9783657791217_011

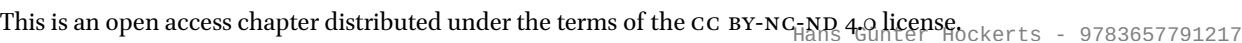


der Kirche und dem NS-Regime. ${ }^{2}$ Aus Anlass der Tagung, die der vorliegende Sammelband dokumentiert, habe ich ein etwas vergilbtes Exemplar hervorgeholt und nach langer Zeit nochmals gelesen - mit der durchaus bangen Frage, ob das, was ich vor einem halben Jahrhundert geschrieben habe, noch valide ist oder ob der Autor im Licht nachträglicher Einsicht in Sack und Asche gehen muss. Für alle Fälle hatte ich mir ein Zitat aus Max Webers „Wissenschaft als Beruf“ zurechtgelegt, das (etwas abgekürzt) lautet: „Wissenschaftlich überholt zu werden, ist nicht nur unser aller Schicksal, sondern unser aller Zweck“. Damit habe „sich jeder abzufinden, der der Wissenschaft dienen will“. ${ }^{3}$

Am Ende der Relektüre stand ein Einerseits/Andererseits. Eine Reihe von Befunden hat nach wie vor Bestand. Doch fällt auch auf, dass ich manche Fragen, die wir heute stellen, damals nicht oder nur ganz am Rande gestellt habe. Es gibt also eine deutliche Differenz zwischen dem Erkenntnisinteresse damals und heute. Die folgende Skizze soll bilanzieren, was Bestand hat (I) und was überholt ist (II). Soweit der Gang der Forschung die ursprünglichen Befunde bekräftigt oder ergänzt hat, wird er in Teil I einbezogen. ${ }^{4}$

Blickt man zunächst auf das Timing der Kampagne, so fällt ein Wechselspiel von Start und Stopp auf. Sie begann im Mai 1936, gestützt auf eine Welle von Strafprozessen. Ende Juli wurde dann beides gestoppt, sowohl die öffentliche Kampagne als auch die Durchführung der Gerichtsverfahren. Die Weisung kam von Hitler selbst - zunächst mit Rücksicht auf die Olympischen Spiele, die im August 1936 in Berlin stattfanden. Im Scheinwerferlicht der Weltöffentlichkeit wollte sich das NS-Regime hochglanzpoliert zeigen, ohne so störende Berichte. Danach blieb die Frage der Fortsetzung eine Weile in der Schwebe, denn Hitler machte einen Test. Er wollte herausfinden, ob die antikommunistische Haltung der Kirche - angestachelt vom spanischen Bürgerkrieg - sich in einen Blankoscheck für das NS-Regime umwandeln lasse. Daher unterbreitete er ein Angebot: Wenn die Kirche sich ohne Wenn und Aber in die antibolschewistische Einheitsfront einreiht, dann werde er ,all das Kleine, was

2 Hans Günter Hockerts, Die Sittlichkeitsprozesse gegen katholische Ordensangehörige und Priester 1936/37. Eine Studie zur nationalsozialistischen Herrschaftstechnik und zum Kirchenkampf. Mainz 1971.

3 Max Weber, Wissenschaft als Beruf [1919], in: ders., Gesammelte Aufsätze zur Wissenschaftslehre, hrsg. von Johannes Winckelmann. Tübingen 1988, 582-613, Zitat 592.

4 Eigens belegt wird im Folgenden nur der weitere Gang der Forschung; im Übrigen sei auf die Belege im genannten Buch (wie Anm. 2) verwiesen. 
die friedliche Zusammenarbeit stört [...] aus der Welt schaffen“. Dabei nannte er ausdrücklich jene Prozesse - so in einem Gespräch mit dem Münchner Kardinal Faulhaber im November 1936. ${ }^{5}$ Der Akzent lag auf ohne Wenn und Aber. Das Angebot zur Verständigung zielte auf einen Unterwerfungsfrieden. ${ }^{6}$

Umso mehr schlug dann die Enzyklika "Mit brennender Sorge“ ein, die im März 1937 in allen katholischen Pfarrkirchen Deutschlands verlesen wurde. Das war ein langer, heute auch langatmig wirkender Text, dessen Kernaussage vom Publikum damals aber sehr gut verstanden wurde: eine Anklage der „versteckten und offenen Verfolgung" der Kirche in Deutschland, wo das Reichskonkordat fortlaufend verletzt werde. Das päpstliche Rundschreiben warnte vor einer Vergötzung von Rasse und Volk und berief sich auf das Naturrecht, wonach "der Mensch als Persönlichkeit gottgegebene Rechte“ besitzt, die keine Gemeinschaft je leugnen oder aufheben dürfe.

Das NS-Regime verstand die Enzyklika als offene Kampfansage und ließ zwölf Druckereien enteignen, die sie gedruckt hatten. Der heftigste Vergeltungsschlag lag jedoch darin, dass die Prozesse und die Kampagne im April 1937 wieder gestartet wurden - mit einem gegenüber dem Vorjahr extrem gesteigerten Agitationsgrad. Man übertreibt nicht, wenn man von einer gewaltigen Aggressionsentladung spricht. Das ließ sich anhand einer Presseauswertung nachweisen. Die zu Beginn der 198oer Jahre zugänglich gewordenen Tagebuchnotizen von Joseph Goebbels geben seine eigene Sicht wieder: Der Chefpropagandist sprach von einem „Höllenkonzert", einem "Großangriff“ mit "gröbstem Geschütz" auf die „schwarze Brut“. Seine eingangs genannte Rede - „2 Stunden in Glanzform“ - empfand er als vollen Erfolg: „Das Publikum rast“. Nachher sei er beim „Führer" gewesen: „Er drückt mir die Hand. Hat die ganze Rede am Rundfunk gehört und, wie er mir sagte, keine Minute still sitzen können “. ${ }^{7}$ - Umgekehrt notierte ein enger Mitarbeiter des Berliner Bischofs Preysing tief bedrückt: „Spätere Generationen werden auch nicht annähernd ermessen können, welche Wirkung das Trommelfeuer der Prozessauswertung der Sittlichkeitsvergehen und -verbrechen hervorrief". ${ }^{8}$

5 Ähnlich in einem Gespräch mit dem Augsburger Weihbischof Eberle im Dezember 1937. Vgl. Gerhard Hetzer, Kulturkampf in Augsburg 1933-1945. Augsburg 1982, 61. Freundlicher Hinweis von PD Dr. Martina Steber.

6 „Wir müssen die Kirchen beugen und sie uns zu Dienern machen“, so fasste Goebbels eine Unterredung mit Hitler im Mai 1937 zusammen. Vgl. Hans Günter Hockerts, Die GoebbelsTagebücher 1932-1941. Eine neue Hauptquelle zur Erforschung der nationalsozialistischen Kirchenpolitik, in: Dieter Albrecht u.a. (Hrsg.), Politik und Konfession. Festschrift für Konrad Repgen zum 6o. Geburtstag. Berlin 1983, 359-392, Zitat 379.

7 Vgl. Hockerts, Goebbels-Tagebücher, 378 .

8 Walther Adolph, Geheime Aufzeichnungen aus dem nationalsozialistischen Kirchenkampf 1935-1943, bearb. von Ulrich von Hehl. Mainz 1979, 126f. (Notiz vom 8.6.1937). 
Wie die Kampagne im Einzelnen gesteuert und kontrolliert wurde, sei hier nicht näher ausgeführt. Es genüge der Hinweis auf eine Kombination von staatlichem Nachrichtenmonopol, verbindlichen "Sprachregelungen“ und obligatorisch abzudruckenden „Auflageberichten“. Nicht alle Zeitungen schlugen dabei so schrille Töne an wie die auflagenstarke Parteipresse. Aber kein einziges Blatt konnte sich ganz entziehen. Dafür sorgten die Berichte, die abgedruckt werden mussten, manchmal zwei oder drei pro Tag.

Das Ziel des agitatorischen Feldzugs lässt sich knapp umreißen. Es ging um einen Machtkampf, in dem das NS-Regime die Kirche diskreditieren und lähmen wollte. So sollte die Gegenwehr gegen die zunehmende Einschnürung ihres gesellschaftlichen Wirkungsraums gebrochen werden, gerade auch auf Feldern, die eigentlich durch das Konkordat geschützt waren, vor allem in der Jugenderziehung, aber auch im Presse- und Verbandswesen. Da die Klöster und Orden den besonderen Argwohn der NS-Ideologen erregten - sie galten als international vernetzte ,Kampftruppe der Kirche -, bezweckte die Kampagne auch, das Ansehen der Ordensgemeinschaften generell zu erschüttern. Der Stempel „Klosterprozesse“, den die gelenkte Presse den Verfahren aufdrückte, verdeutlicht diese Stoßrichtung.

Aber siehe da:Ende Juli 1937 wurde die Kampagne abermals gestoppt, ebenso die laufenden Strafverfahren. Die Weisung kam von ganz oben. Einige Monate später erläuterte Hitler in einer Konferenz mit den militärischen Spitzen der Wehrmacht, er sei entschlossen, in den nächsten Jahren die deutsche Raumfrage zu lösen - auch mit Gewalt. Hier wird ein Zusammenhang erkennbar: Der Diktator war schlau genug, um außen- und innenpolitische Konflikte nicht gleichzeitig zu schüren. Also fuhr er den Kirchenkonflikt herunter, als er außenpolitisch auf Konfliktkurs mit Kriegsrisiko ging. So hatte ich den Umschwung in meinem Buch erklärt. Die Goebbels-Tagebücher erlauben indes eine Präzisierung. Demnach war es der Propagandaminister, der den „Führer“ Ende Juli für einen Stopp der Kampagne gewann, damit „das Volk nicht dagegen abstumpft“. Dabei war zunächst nur an eine vorübergehende Pause gedacht. Noch im Dezember 1937 notierte Goebbels, Hitler sei bereit, die „Pfaffenprozesse zu gegebener Zeit wieder anzudrehen“, aber - und hier trat nun die außenpolitische Priorität deutlich nach vorn - einstweilen wolle er „Ruhe" in der Kirchenfrage. ${ }^{9}$

Es blieb fortan bei der Linie des gedämpften Kirchenkonflikts. Die Einschnürungspolitik ging zwar weiter, wie die Enteignung zahlreicher Klöster und kirchlicher Einrichtungen 1940/41 verdeutlicht, nun aber zumeist unter

9 Vgl. Hockerts, Goebbels-Tagebücher, 379f. 
dem Deckmantel kriegsbedingter Notwendigkeiten. ${ }^{10}$ Die sistierten Prozesse wurden nach und nach wieder aufgenommen, aber anscheinend nicht alle und nicht mehr mit Propagandalärm. Wie eine neuere Studie zeigt, gab es in Österreich nach dem „Anschluss“ eine Reihe von Prozessen, die sich „durchaus mit jenen im Altreich in den Jahren 1936/37 vergleichen“ ließen, doch ohne vergleichbare Propagandaoffensive." In diesem Zusammenhang ist die feierliche Loyalitätserklärung bemerkenswert, mit der die österreichischen Bischöfe den „Anschluss“ begrüßten: Im Geflecht der Motive spielte anscheinend auch die Furcht eine Rolle, in Österreich könne sonst eine ähnliche Sittlichkeitsprozess-Kampagne drohen wie zuvor im Reichsgebiet. ${ }^{12}$

Wenden wir uns nun der juristischen Seite zu. Wie verliefen die Ermittlungsund Hauptverfahren? Haben die Gerichte nach der damaligen Rechtslage korrekt entschieden? Zunächst zu den Ermittlungen. Seit dem Herbst 1935 führte ein Sonderkommando der Gestapo große Razzien in kirchlichen Einrichtungen durch. Dem „Dezernat zur Bearbeitung von homosexuellen Fällen“ im Berliner Geheimen Staatspolizeiamt unterstellt, durchkämmte es mit bis zu $5^{\circ}$ Beamten mehrere Laienkongregationen in den Bistümern Trier und Köln. ${ }^{13}$ Die Laienbrüder waren in der Krankenpflege und der Fürsorgeerziehung tätig, auch in Anstalten für geistig behinderte Menschen. Später durchkämmte das Kommando weitere kirchliche Kreise und andere Regionen; es durchsuchte auch mehrere Generalvikariate, im Fall des Trierer Bischofs Bornewasser auch dessen Wohnräume.

Das Gestapo-Kommando hatte nach geltendem Recht den Status von „Hilfsbeamten“ der örtlichen Staatsanwaltschaft. Doch galt das nur, soweit die Gestapo bereit war, sich an die Strafprozessordnung zu halten. Sie konnte es auch vorziehen, eigenmächtig zu handeln - vor allem mit der Androhung oder Verhängung von "Schutzhaft“. Tatsächlich verfuhr das Kommando - geleitet vom Chef des besagten Berliner Dezernats, Kriminalkommissar Gerhard Kanthack - selbstherrlich und rabiat. Eine neuere biographische Untersuchung

10 Vgl. Annette Mertens, Himmlers Klostersturm. Der Angriff auf katholische Einrichtungen im Zweiten Weltkrieg und die Wiedergutmachung nach 1945. Paderborn u.a. 2006.

11 Vgl. Albert Knoll/Thomas Brüstle, Verfolgung von Homosexuellen in Oberösterreich in der NS-Zeit, in: Reichsgau Oberdonau, Aspekte 2. (Oberösterreich in der Zeit des Nationalsozialismus, hrsg. vom Oberösterreichischen Landesarchiv, Bd. 4). Linz 2005, 149-203, Zitat 160.

12 Vgl. ein Schreiben des Salzburger Erzbischofs Waitz an den Klerus, 25.3.1938, in: Akten deutscher Bischöfe, 544 .

13 Es handelte sich hauptsächlich um die (mit dem Priesterorden der Franziskaner nicht in Verbindung stehende) Laienkongregation der Franziskaner (Mutterhaus Walbreitbach), die Genossenschaft der Barmherzigen Brüder (Mutterhäuser Montabaur, Trier) und die Alexianerbrüder (Mutterhaus Aachen). 
wirft ein bezeichnendes Licht auf sein Profil: Er fiel 1938 in Ungnade, erhielt eine Gefängnisstrafe, und in einem weiteren Verfahren hob die Staatsanwaltschaft im September 1943 hervor: „Während seiner dienstlichen Tätigkeit als Kriminal-Kommissar war Kanthack als besonders rücksichtsloser und brutaler Beamter bekannt; er führte den Beinamen ,Chef der Tscheka', wie er selbst rühmend hervorgehoben hat. ${ }^{114}$

Der Sicherheitsdienst der SS (SD) beteiligte sich ebenfalls an den Nachforschungen. Das hatte ich aufgrund einiger Indizien vermutet; inzwischen weiß man mehr. Auf Sachakten aus dem SD-Hauptamt gestützt, die nach dem Fall der Mauer zugänglich wurden, hat Wolfgang Dierker den Wirkungsanteil dieser Überwachungsinstanz genauer herausgearbeitet, auch deren operative Vorschläge zur Durchführung der Kampagne. Es ist bemerkenswert, dass einige SD-Mitarbeiter durchaus nicht mit Kritik an Ungereimtheiten und Übersteigerungen in der propagandistischen Ausbeutung sparten: Das gehe auf Kosten der Glaubwürdigkeit.15

Im Hinblick auf die Staatsanwaltschaft traf Reichsjustizminister Gürtner Ende 1935 eine Sonderregelung. Speziell für die Verfahren gegen die hauptbetroffenen Laienkongregationen richtete er eine Zweigstelle Koblenz der Zentralstaatsanwaltschaft - einer ihm unmittelbar unterstellten Behörde ein. Dabei gaben zwei Motive den Ausschlag. Erstens kamen Ermittlungsverfahren gegen Laienbrüder in so großer Zahl in Gang, dass die örtlich zuständige Staatsanwaltschaft überlastet gewesen wäre. Zweitens gewann die Justiz somit mehr Gewicht gegenüber dem Gestapo-Sonderkommando, das sich zuvor als Herr der Verfahren aufgespielt hatte. Gemäß der bekannten DoppelstaatsThese von Ernst Fraenkel kann man hier Spannungen zwischen Instanzen des "Maßnahmenstaats" (Gestapo) und des "Normenstaats" (Staatsanwaltschaft) erkennen. Doch nahmen die Friktionen ab, als die Kommandoführung im Februar 1936 wechselte: Unter Kanthacks Nachfolger pendelte sich ein Kooperationsverhältnis ein. Dass die Sonderstaatsanwaltschaft eine „normenstaatliche“ Linie repräsentierte, ist in meinem Buch zu Recht gesagt. Doch bekannte sie sich auch loyal zu den "Grundlagen des völkischen Staates“"16 und

14 Vgl. Jens Dobler, Täteropfer. Der Berliner Kriminalkommissar Gerhard Kanthack im KZ Mauthausen, in: Jahrbuch Mauthausen 9, 2015, Wien 2016, 57-68, Zitat 63. - Auf rigide Ermittlungsmethoden der Würzburger Gestapo (mit mindestens einem belegbaren Fall erzwungener Falschaussage) im Kontext der Kampagne verweist Burkhard Jellonnek, Homosexuelle unterm Hakenkreuz. Die Verfolgung von Homosexuellen im Dritten Reich. Paderborn 1990, 243-246.

15 Vgl. Wolfgang Dierker, Himmlers Glaubenskrieger. Der Sicherheitsdienst der SS und seine Religionspolitik 1933-1941. Paderborn u.a. 2002, 178-183.

16 Hockerts, Sittlichkeitsprozesse, 46 Anm. 36o. 
trug auf ihre Weise zur Funktionsfähigkeit der Diktatur bei. Diesen Aspekt würde ich heute stärker gewichten.

Für die Anklagen der Sonderstaatsanwälte waren die Landgerichte Koblenz und Bonn zuständig, somit für die Hauptmasse der Prozesswelle. Sie hatten in den Hauptverfahren oft zu entscheiden, ob allein der Strafrechtsparagraph 175 oder auch 174 zutraf. Um dies kurz zu erläutern: § 175 machte homosexuelle Handlungen zwischen Männern strafbar. 1871 eingeführt, war er 1935 verschärft worden; auch zuvor nicht kriminalisierte Handlungen im einvernehmlichen Umgang waren seither unter Strafe gestellt. § 174 betraf hingegen - unverändert seit 1871 - den sexuellen Missbrauch von Schutzbefohlenen, insbesondere von minderjährigen Schülern oder Zöglingen und den Missbrauch von Patienten in der Krankenpflege. Einem Bericht des Vorsitzenden der Koblenzer Strafkammer zufolge ging es bei der juristischen Beurteilung "fast stets nur" um diese beiden Paragraphen. Selten scheint also auch $\S 176$ relevant gewesen zu sein, der für den Missbrauch von Kindern (Personen unter 14 Jahren) ein besonders hohes Strafmaß vorsah.

Sind die Hauptverfahren nach damaligem Recht korrekt verlaufen? Waren die Urteile - so gesehen - rechtmäßig? Soweit es sich um die Arbeit der genannten Landgerichte handelt, lautet die Antwort: im Kern ja. Die Einschränkung bezieht sich vor allem darauf, dass nicht die Strafprozessordnung, sondern das politische Diktat Hitlers bestimmte, ob und wann es zur Hauptverhandlung kam. Das war ein klarer Verstoß gegen das geltende Recht, auch zu Lasten von Beschuldigten, deren Untersuchungshaft sich unabsehbar verlängerte. Außerdem wurden die Landgerichte mehr oder weniger gezwungen, ein propagandistisch brauchbares Publikum auch bei nicht öffentlichen Teilen der Verhandlungen zuzulassen. Nach einigem Zögern gab der Koblenzer Landgerichtspräsident im Mai 1937 auch die Erlaubnis, eine Reihe von Hauptverhandlungen auf Schallplatten aufzunehmen. Er fand sich mit der Zusicherung ab, dass sie nur in eng begrenzten Kreisen verwendet würden. In Wirklichkeit spielte das Goebbels-Ministerium mit dem Gedanken, die Aufnahmen in die Rundfunkpropaganda einzuspeisen (wozu es dann jedoch nicht kam).

In ihren Urteilen haben die beiden Kammern sich jedoch an das geltende Recht gehalten. Ein aus propagandistischen Gründen ausgesprochenes Fehlurteil konnte ich nicht entdecken, wohl aber Fälle, in denen die Richter durchaus den Grundsatz in dubio pro reo gelten ließen. In einigen strittigen Punkten der Gesetzesauslegung wählten die Richter erst nach Revisionsurteilen des Reichsgerichts die für die Angeklagten weniger günstige Variante. Sogar auf dem Höhepunkt der Kampagne kam es bei unzulänglicher Beweislage zu Freisprüchen. Inwieweit diese Befunde auch für die an anderen Landgerichten durchgeführten Strafverfahren gegen Ordensleute und Geistliche gelten, ist 
weniger sicher. Doch bleibt festzuhalten, dass die kirchliche Gegenwehr zwar die propagandistische Betätigung einiger Staatsanwälte beanstandet hat, aber nicht die Urteile.

Wie sieht nun das quantitative Ergebnis aus? Bezogen auf den Zeitraum von Januar 1933 bis Juli 1937 ergibt sich das folgende Zahlenbild. Verurteilt wurden 57 Weltgeistliche, 7 Ordensgeistliche und 4 Laienbrüder, die einem Priesterorden angehörten, sowie 170 Mitglieder von Laienbrüdergenossenschaften (davon 62 ehemalige). Es schwebten noch Verfahren gegen 47 Weltund Ordensgeistliche sowie gegen rund 200 Laienbrüder (etwa zur Hälfte ehemalige). Ob bei all diesen schwebenden Verfahren eine Verurteilung fällig war, ist ungewiss; immerhin hatte es auch bisher schon etliche Freisprüche und eingestellte Verfahren gegeben. ${ }^{17}$ Aber wenn man die schwebenden Fälle kurzerhand zur Zahl der Verurteilten addiert, um den möglichen Höchstwert der Prozesswelle zu markieren, dann kommt man auf rund 480. Keine geringe Zahl, jedoch nicht jene Tausend oder gar Abertausend, auf die sich die Medienpropaganda eingeschossen hatte. Außerdem sticht hervor, dass es sich zum Großteil um Laienbrüder handelte. Das hatte die Kampagne mit Begriffen wie „kirchliche Sexualverbrecher" verwischt, um auch den Klerus möglichst stark zu belasten.

Diese Zahlen stützen sich (mit einer gleich zu erläuternden Ausnahme) auf eine detaillierte statistische Erhebung, die der Berliner Bischof Preysing mit Hilfe aller Ordinariate durchführte und - um größtmögliche Genauigkeit bemüht - einer Eingabe an den Reichsjustizminister beifügte. Demnach macht die Zahl der Verurteilten, ergänzt um die schwebenden Fälle und bezogen auf die jeweilige Gesamtzahl der Geistlichen beim Weltklerus o,44 Prozent, beim Ordensklerus o,41 Prozent aus. Während der Bischof sich beim Klerus seiner Zahlen sicher war, fügte er bei den Laienbrüdern einen Vorbehalt ein. Wegen des hohen Anteils entlassener oder ausgeschiedener Brüder verfügte er hier über einen weniger verlässlichen Datensatz. Meine Überprüfung ergab, dass er die Zahl der verurteilten Laienbrüder treffend wiedergab, jedoch die Zahl der noch schwebenden Verfahren - somit auch den Prozentsatz mit 7,9 - zu niedrig ansetzte. ${ }^{18}$

\footnotetext{
17 Bei Welt- und Ordensgeistlichen 21; bei Laienbrüdern (soweit es sich um die Prozesse im Bereich der Sonderstaatsanwaltschaft handelte) 19. Ohne die Verschärfung des $§ 175 \mathrm{im}$ Jahr 1935 wäre die Zahl der Freisprüche höher ausgefallen.

18 Meine Überprüfung bezog sich auf den Bestand der Anklagen und Urteile im Bereich der Sonderstaatsanwaltschaft. Demnach schwebten nicht nur 66 Verfahren, wie Preysing errechnete, wobei er anscheinend nur die noch unerledigten Anklagen berücksichtigte. Bezieht man auch die laufenden Vorverfahren (Ermittlungen) ein, kommt man auf etwa 200.
} 
Preysings Zahlenwerk differenzierte nicht zwischen den Fällen der Paragraphen 174, 175 und 176, obwohl der Unterschied sehr gravierend ist. Beim $§ 175$ ging es um einvernehmliches Verhalten von Erwachsenen, in den anderen Fällen jedoch um sexuellen Missbrauch von Minderjährigen und Schutzbefohlenen - also eine ganz andere Kategorie, ein Verbrechen, zumal dann, wenn damit die Androhung oder Anwendung von Gewalt verbunden war. Dass die Statistik diese Unterscheidung nicht traf, sondern die Paragraphen gewissermaßen nivellierte, lässt kritische Rückfragen zu, die mir damals nicht in den Sinn gekommen sind. ${ }^{19}$ Zwar ist meinem Buch zu entnehmen, dass es beim Großteil der in Koblenz und Bonn verhandelten Fälle gerade auch um den Paragraphen 174 ging, aber im Nachhinein vermisse ich eine numerische Präzisierung, auch mit Blick auf das Alter der Missbrauchten.

Preysing war der für Pressefragen zuständige Bischof im Kreis des deutschen Episkopats. Daher übernahm er es, Informationen zu sammeln und als Sprecher der Amtskirche gegenüber der Reichsregierung aufzutreten. Im Mai 1937 wandte er sich mit einer Protesteingabe an den Propagandaminister. Dabei griff er die "Prozesse als solche" nicht an, umso mehr jedoch die Methoden der propagandistischen Ausschlachtung, die „Aussaat des Hasses“, deren Radikalität den Willen zum „Vernichtungskampf gegen die katholische Kirche“ anzeige. Auch in seiner Eingabe an den Reichsjustizminister unterschied Preysing zwischen den „Prozessen an sich“ und dem Propagandafeldzug. Dieser sei, so betonte er abermals, ein Vorstoß „im augenblicklichen Stadium des Vernichtungskampfes gegen die katholische Kirche in Deutschland“.

Irgendein Erfolg war den Eingaben an die Reichsregierung nicht beschieden. Die Bischöfe hatten auch keinerlei Chance, in den gelenkten Medien zu Wort zu kommen. Umso größeren Wert legten sie auf die Binnenkommunikation mit dem Kirchenvolk, um den Keil des Misstrauens abzuwehren, den die Kampagne zwischen das katholische Bevölkerungsdrittel und die kirchlichen Institutionen treiben wollte. Ihre Hirtenbriefe durften zwar nicht in den Kirchenzeitungen abgedruckt werden (so weit reichten die Repressalien des Regimes), sie wurden aber von den Kanzeln verlesen - bei damals vollen Kirchen. Der Tenor war: Bestürzung und Beklagen der Delikte einerseits; Kritik an der Art und den Absichten der medialen Ausbeutung andererseits (wobei nicht alle Bischöfe einen so kämpferischen Ton anschlugen wie Preysing), verbunden mit dem Appell, sich in dieser Zeit der Gefährdung gerade nicht

19 Dabei ist allerdings auch der Kontext zu beachten: Die Eingabe wollte mit dieser Statistik „die unwahre Zahlenpropaganda“ widerlegen, die mit der Tausenderzahl operierte. Es wäre zu prüfen, ob die von den Ordinariaten an Preysing gelieferten Daten eine Differenzierung vornahmen. 
von den Kirchengegnern entzweien zu lassen. ${ }^{20}$ Der Trierer Bischof, in dessen Diözese sich die Verfahren gegen Laienbrüder auffällig häuften, verwies darauf, dass er mit besonderen Vollmachten eine gründliche Visitation aller in Betracht kommenden Ordenshäuser eingeleitet habe..$^{21}$ Und er gestand ein, dass er im Falle eines wiederholt rückfälligen Pfarrers (Vergehen an Jugendlichen) eine "Milde und Langmut" habe walten lassen, die "für die Zukunft nicht mehr am Platze" sei.

Gelang es den Bischöfen, eine innerkirchliche Vertrauenskrise abzuwehren? Dagegen könnte der sprunghafte Anstieg der Kirchenaustrittszahlen sprechen: 1937 verließen 108 ooo Katholiken die Kirche, ein Spitzenwert, der in keinem anderen Jahr der Weimarer Republik und der NS-Zeit auch nur annähernd erreicht wurde. Aber auf der evangelischen Seite, die von der Kampagne unberührt blieb, schnellte die Austrittskurve 1937 sogar noch stärker nach oben. Daher ist zu vermuten, dass wohl weniger das Thema „Sittlichkeitsprozesse“ als vielmehr der generelle Druck auf die Kirchen den Ausschlag gab, der in dieser Phase der NS-Religionspolitik massiv gesteigert wurde. Außerdem machte jener Spitzenwert, gemessen an der Gesamtzahl der Katholiken, nur o,48 Prozent aus, blieb also ein Randphänomen. Es ist methodisch sehr schwierig, die Resonanz der Kampagne präzise zu erfassen, doch deutet eine Reihe von Indikatoren darauf hin, dass die Agitation jedenfalls in den Kernzonen des katholischen Milieus nicht verfing. Habituelle Dispositionen lassen sich nicht so schnell ändern. Außerdem trug der so grobe und offensichtlich tendenziöse Zuschnitt der Offensive dazu bei, dass im katholischen Kernmilieu eher die gelenkten Medien als die Autorität der Bischöfe in Misskredit gerieten.

\section{II.}

Als das hier wieder zur Hand genommene Buch entstand, gab es noch keine Historiographie zur Verfolgung von Homosexuellen in der NS-Zeit. Diese Forschungsetzte erst in den197oerJahre ein und führte nochlange ein "Schattendasein“.22 Das hat sich inzwischen geändert. Heute gehört es zum gesicherten

20 Auf eine Besonderheit im Bistum Augsburg weist Hetzer, Kulturkampf, 59f. hin: Bischof Kumpfmüller regte eine Sühneandacht in den Pfarreien zur Buße für die Missbrauchstaten an. Ein erheblicher Teil der Pfarrer erklärte sich jedoch „schroff dagegen“, weil diese Geste als Einknicken vor der Propagandaoffensive verstanden werden konnte.

21 Besondere Vollmachten waren bei Laiengenossenschaften päpstlichen Rechts erforderlich, da sie nur begrenzt der bischöflichen Aufsicht unterstanden.

22 Alexander Zinn, „Aus dem Volkskörper entfernt“? Homosexuelle Männer im Nationalsozialismus. Frankfurt a. M., 13. - Zu den Wegmarken der Forschung zählen Jellonnek, 
Wissen, dass die Verfolgung homosexueller Männer ab 1935 generell (also nicht nur mit Blick auf Klöster und Priester) sehr verschärft wurde - gemäß der sozialbiologischen Doktrin, dass der "Volkskörper" sonst "verseucht" und "zersetzt“ werde. Dabei trat Himmler als treibende Kraft hervor, zumal ihn noch eine weitere Obsession plagte: In der Natur von Homosexuellen liege ein für die Staatsmacht gefährlicher Hang zu Seilschaften und Verrat. ${ }^{23}$ So leitete Himmler 1935/36 eine „Generalmobilmachung gegen Homosexuelle“24 ein. Dazu gehörte die Entsendung von Gestapo-Sonderkommandos in eine Reihe von Städten, um gegen die lokale Homosexuellenszene vorzugehen.

Vor diesem Hintergrund hat Alexander Zinn argumentiert, die Sittlichkeitsprozesse gegen Ordensleute und Priester müssten mehr in diesen allgemeinen Rahmen eingeordnet werden. Dann werde auch deutlich, dass die genuin homophobe Motivation der Verfolgungswelle in meinem Buch unterschätzt werde. Dort sei zu sehr das taktische Interesse an einer kirchenpolitisch instrumentalisierbaren Waffe als treibendes Motiv betont. ${ }^{25}$ Eine geheime Programmrede, die Himmler im Februar 1937 vor SS-Führern zum Thema Homosexualität hielt, unterstützt diesen Einwand: Der zum Chef der deutschen Polizei aufgestiegene „Reichsführer SS“ verknüpfte die Klosterund Sittlichkeitsprozesse ausdrücklich mit dem primär homophoben Feindbild. Die Prozesse sollten demnach den schlüssigen Beweis erbringen, „dass die Kirchenorganisation in ihrer Führerschaft, ihrem Priestertum, zum überwiegenden Teil ein homosexueller erotischer Männerbund“ sei, der „auf dieser Grundlage seit nunmehr 1800 Jahren die Menschheit terrorisiert", und dabei wie schon in den Hexen- und Ketzerprozessen - „sadistisch pervers“ vorgehe. Himmler hielt zwar die Mehrheit der Landpfarrer nicht für homosexuell, doch in den Klöstern schätzte er den Anteil schnurstracks auf 90 bis 100 Prozent. ${ }^{26}$

Homosexuelle und Günter Grau (Hrsg.), Homosexualität in der NS-Zeit. Dokumente einer Diskriminierung und Verfolgung. Frankfurt a. M. 1993.

23 Das „Stereotyp von gefährlich-verräterischen homosexuellen Seilschaften“ war keine Erfindung der NS-Diktatur, doch wirkte diese als „Brandbeschleuniger“, vgl. Michael Schwartz, Homosexuelle, Seilschaften, Verrat. Ein transnationales Stereotyp im 20. Jahrhundert. Berlin 2019, Zitat 12. Schwartz verweist auch darauf, dass die NS-Kampagne „weit zurückreichende diskursive und repressive Vorläufer" hatte. So seien z.B. auch im preußischen bzw. reichsdeutschen „Kulturkampf“ der 186oer bis 188oer Jahre skandalisierungsfähige Fälle sexueller Vergehen als kirchenpolitische Waffe eingesetzt worden (S. 203).

24 Zinn, „Volkskörper“, 295.

25 Ebd., 33 .

26 Erstmals publiziert in: Bradley F. Smith/Agnes F. Peterson (Hrsg.), Heinrich Himmler. Geheimreden 1933 bis 1945 und andere Ansprachen. Frankfurt a. M. 1974, 93-104, Zitat 
Zinns Einwand ist also berechtigt. Der übergreifende Zusammenhang der in meinem Buch untersuchten Gestapo-Aktionen ist nun besser erkennbar. Das ändert allerdings nichts daran, dass die Prozesse gegen Ordensleute und Priester in ganz besonderer Weise politisch instrumentalisiert und propagandistisch ausgebeutet wurden. Das machttaktische Kalkül beherrschte die Kampagne so offensichtlich, dass die Prozesswelle nicht allein im Kontext der generell verschärften Homosexuellenverfolgung zu sehen ist. Die aktuellen Spannungen zwischen Kirche und Regime kamen als treibender Faktor eigener Art hinzu.

Weiter reicht ein anderer Einwand, der einen Wandel im Zuschnitt von Fragestellungen und Beurteilungskriterien anzeigt: Es sei an der Zeit, die Prozesse nicht mehr nur oder primär im Paradigma des NS-Kirchenkampfs zu betrachten, sondern auch unter Gesichtspunkten der Diskriminierung von Homosexuellen, der Geschichte des sexuellen Missbrauchs und des kircheninternen Umgangs mit Tätern und Opfern. ${ }^{27}$

Damit sind zweifellos weiterführende Perspektiven angesprochen. Wichtig ist dabei die kategoriale Unterscheidung zwischen den Sachverhalten, auf die sich die Strafrechtsparagraphen bezogen. Wer wegen gleichgeschlechtlicher Beziehungen ( $(175)$ verurteilt wurde, beging nach unserem heutigen Verständnis gerade keine kriminelle Handlung, sondern zählt - zumal dann, wenn KZHaft die Folge war - zu den Verfolgten des NS-Regimes. Der kulturelle und juristische Deutungsrahmen hat sich so grundlegend gewandelt, wenn auch langsam und für die Betroffenen mühsam, dass jener Paragraph 1969 und 1973 entschärft und 1994 ersatzlos gestrichen wurde. Der Bundestag hat die in der NS-Zeit ergangenen Urteile nach $§ 175 \mathrm{im} \mathrm{Jahr} 2002$ als Unrecht qualifiziert und förmlich aufgehoben. Vor diesem Hintergrund kommt die Kirche als „Träger traditioneller Homosexuellen-Diskriminierung " in den Blick. ${ }^{28}$ So gewendet, rücken die Sittlichkeitsprozesse in ein anderes Licht und werden Teil einer Geschichte der theologisch bzw. kirchenamtlich gestützten Homophobie.

Allerdings wäre es verfehlt, diesen Teil für das Ganze zu nehmen. ${ }^{29}$ Denn es ging in der Prozesswelle ja auch - sogar großenteils - um den sexuellen

102f. Auf diese Schlüsselquelle bezieht sich auch Peter Longerich, Heinrich Himmler. Biographie. München 2010, 242-247.

27 So argumentieren z.B. Michael Schwartz (S. 13f.) und Stefanie Wolter (S. 55f.) in: Michael Schwartz (Hrsg.), Homosexuelle im Nationalsozialismus. Neue Forschungsperspektiven zu Lebenssituationen von lesbischen, schwulen, bi-, trans- und intersexuellen Menschen 1933 bis 1945. München 2014 .

28 Ebd., 13.

29 So bei Detlev Müller/Jürgen Müller, „Dienstags gesündigt, mittwochs gebeichtet“. Die Sittlichkeitsprozesse gegen die katholische Kirche in den Jahren 1936/37, in: Cornelia Limpricht/Jürgen Müller/Nina Oxenius (Hrsg.), ,Verführte“ Männer. Das Leben der Kölner Homosexuellen im Dritten Reich. Köln 1991, 76-81. 
Missbrauch von Minderjährigen und Schutzbefohlenen. In dem nun wieder gelesenen Buch hatte ich die öffentliche Reaktion des Episkopats untersucht, jedoch den kircheninternen Umgang mit dem Missbrauchsgeschehen nur gestreift: Die von Bischof Bornewasser eingeleitete Visitation brachte bei den Waldbreitbacher Laienbrüdern so schlimme Vorfälle und Zustände ans Licht, dass er in Rom die Auflösung dieser Genossenschaft beantragte, zu der es dann auch kam. ${ }^{30}$ Eine weitere Initiative betraf den Rechtsstatus der Laienkongregationen, die als Genossenschaften päpstlichen Rechts nur begrenzt der bischöflichen Aufsicht unterlagen. Von der Häufung der Delikte aufgeschreckt, regte die deutsche Bischofskonferenz in Rom eine Neuregelung an, die dem jeweiligen Ortsordinarius mehr Kontrolle und Eingriffe ermöglichen sollte. Den "Waldbreitbacher Skandal“ nahm die Konferenz ferner zum Anlass für ein Schreiben an sämtliche Ordensleitungen und Vorstände der selbständigen Klöster in Deutschland. Darin ermahnte sie, alles zu tun, damit "solche und ähnliche Ärgernisse in Zukunft nicht mehr vorkommen“. Geboten sei ein „wachsames Auge“.31

Aber wie wachsam war das Auge im eigenen Verantwortungsbereich der Bischöfe? Das Skandaljahr 2010 und die aktuellen Aufarbeitungsprojekte zum Umgang der Bistumsleitungen mit Fällen sexualisierter Gewalt verändern auch den historischen Blickwinkel und rücken Fragen wie diese nach vorn: Wer wusste was und wie reagierten die Verantwortlichen? Wurde systematisch vertuscht? Was verbirgt sich hinter Chiffren wie "Milde und Langmut", die sich nicht allein im Wortschatz Bornewassers finden? Zu fragen ist auch, ob die Ordinariate aus der doch schockartigen Erfahrung der Prozesswelle Konsequenzen für das eigene Aufsichts- und Präventionsverhalten gezogen haben. Und mit Blick auf einen größeren Zeitbogen: Wie hat die Erinnerung an die Sittlichkeitsprozesse später, in der Bundesrepublik, das Verhalten der Bistumsleitungen beeinflusst? Anscheinend lebte zumeist nur die kirchenfeindliche Instrumentalisierung in der Erinnerung fort, während das Missbrauchsgeschehen weithin aus dem Gedächtnis verschwand. Daher spricht einiges für die Annahme, dass die Erinnerung geradezu kontraproduktiv wirkte: Demnach hat sie gerade nicht für das Thema „sexueller Missbrauch“

30 Die Visitation hatte neben krassem Aufsichtsversagen auch ergeben, dass zur Zeit der Massenarbeitslosigkeit viele Personen allein aus materieller Not in die Laienkongregationen geströmt waren. Von übersteigertem Expansionsdrang getrieben, seien die Ordensoberen bei der Auswahl viel zu leichtfertig vorgegangen.

31 Das in der Bischofskonferenz am 20.8.1936 beschlossene Schreiben ist ediert in: Bernhard Stasiewski (Hrsg.), Akten deutscher Bischöfe über die Lage der Kirche 1933-1945, Bd. III: 1935-1936. Mainz 1979, 457f. 
sensibilisiert, vielmehr die Bereitschaft verstärkt, die Aufdeckung von Missbrauchsfällen als Attacke auf das Ansehen der Kirche abzuwehren.

Während der Plenarkonferenz des deutschen Episkopats im August 1937 stellte Bischof Galen einige eindringliche Fragen: „Was sollen wir dagegen machen, damit in Zukunft derartige Zustände nicht wiederholt werden? Wie können die Angeklagten und Verurteilten untergebracht werden?" Und nochmals: „Was soll mit den gefallenen Priestern geschehen?“32 In einer Denkschrift zur Lage der Kirche, die er im Vorfeld der Konferenz verfasste, hob Galen das medial geschürte "Empfinden“ hervor, dass es "an Wachsamkeit und Strenge seitens der zuständigen kirchlichen Oberer gefehlt“ habe, und er mahnte: „Ob und in welcher Form es möglich ist, in dieser Hinsicht wirkliche Aufklärung und die Zusicherung vermehrter Vorbeugungsmaßnahmen zu geben, bedarf ernstester Überlegungen. Sie wären jedenfalls dringend erwünscht!" ${ }^{\text {“3 }}$ Den bisher edierten Akten lässt sich nicht entnehmen, ob Galens Appell, der sonderbar aktuell klingt, eine interne Debatte ausgelöst hat. ${ }^{34}$ Ein langfristig wirksamer Lernprozess scheint jedenfalls nicht in Gang gekommen zu sein.

Bei genauerem Hinsehen weist Galens Besorgnis eine merkwürdige Lücke auf: Wie man den Opfern des sexuellen Missbrauchs helfen könne, fragte er nicht. Das entspricht einem Befund aktueller Aufarbeitungsprojekte, wonach die Fürsorge von Bistumsleitungen lange weitaus mehr den Beschuldigten oder auch Tätern galt als den Opfern. Die Betroffenen fanden oft kaum oder gar keine Beachtung. Ein historiographisches Desiderat ist es jedoch, die Leidensund Erfahrungsgeschichte der Betroffenen und den Umgang der kirchlichen Instanzen mit ihnen möglichst ans Licht zu bringen. Thomas Schnitzler hat im Bistumsarchiv Trier einen einschlägigen Bestand entdeckt und 2011 mit einer Sondererlaubnis auswerten dürfen. Darin ermittelte er die Namen von 300 Kindern und Jugendlichen, die in der NS-Zeit in Pflegeheimen des Bistums Trier missbraucht worden sind. Seine Studie wirft Licht auf die Gelegenheitsstruktur sexualisierter Gewalt, kann aber noch nicht viel über den biographischen Weg

32 Vgl. die Mitschrift des Speyrer Bischofs Sebastian, die mehr besagt als das kursorische Protokoll, in: Akten deutscher Bischöfe, 331.

33 Vgl. die Denkschrift vom 31.7.1937, in: Bischof Clemens August Graf von Galen. Akten, Briefe und Predigten 1933-1946, Bd. I: 1933-1939, bearb. von Peter Löffler, 2. erw. Aufl. Paderborn 1996, 552-556, Zitat 553. Der Empfängerkreis ist dort (mit Ausnahme des Münchner Kardinals Faulhaber) nicht ausgewiesen.

34 Zu beachten sind erhebliche Differenzen in und zwischen den einzelnen Diözesen. Der Breslauer Kardinal Bertram galt im Unterschied zu anderen Bischöfen als „unnachgiebig hart in der Ahndung sittlicher Vergehen seiner Geistlichen“. Im Bistum Berlin bildete die Ahndungsfrage sogar einen „Streitpunkt“ zwischen dem Generalvikar („weitherzig“) und seinem Bischof („rigoros“). Vgl. Adolph, Geheime Aufzeichnungen, 94 und 126. 
der Betroffenen sagen. ${ }^{35}$ Bei dieser Kategorie von Missbrauchsopfern war die Gefahr groß, auch in die Fänge der Zwangssterilisierung und schließlich in das Tötungsprogramm der nationalsozialistischen „Euthanasie“ zu geraten. ${ }^{36}$

Zurück zu meinem Buch von 1971. Damals konnte ich mich über ein positives Echo freuen. Keine Besprechung übte Kritik an der Wahl der Fragestellungen oder der Gewichtung der Ergebnisse. Sogar der Spiegel nicht, der sich in einem ziemlich langen Bericht eng an die Befunde des Buches hielt. ${ }^{37}$ Auch eine Rezension in The American Historical Review interessierte sich für genau die Problemkreise, die im Fokus der Studie stehen: die nationalsozialistische Herrschaftstechnik, die Rolle der Justiz und der Konflikt zwischen Hitlers Regime und der katholischen Kirche. ${ }^{38}$ Seither sind jedoch - das zeigt die Relektüre nach 50 Jahren - neue Perspektiven hinzugekommen, die einen zweiten Blick auf die Prozesse gegen Ordensleute und Priester in der NS-Zeit ermöglichen, und die das eingangs zitierte Diktum Max Webers wenn nicht ganz, so doch zum Teil bestätigen: „Wissenschaftlich überholt zu werden, ist nicht nur unser aller Schicksal, sondern unser aller Zweck“.

35 Thomas Schnitzler, Sexualisierte Gewalt in Pflegeheimen des Bistums Trier. Unbekannte Vorfälle vor 1945, Vortrag vom 9.5.2015, einzusehen unter https://www.stiftungdemokratie-saarland.de/fileadmin/62sds-web/medien/news/Vortraege_PDF/Sexuelle_ Gewalt_in_Pflegeheimen.pdf, zuletzt aufgerufen am 21.9.2020.

36 Eine solche biographische Verkettung könnte am Beispiel der Heil- und Pflegeanstalt der Barmherzigen Brüder in Trier überprüft werden, die einerseits in dem von Schnitzler ausgewerteten Bestand erfasst ist, andererseits auch bei Matthias Klein, NS-„Rassenhygiene“ im Raum Trier. Zwangssterilisationen und Patientenmorde im ehemaligen Regierungsbezirk Trier 1933-1945. Wien u.a. 2020. Der Weg von der Trierer Anstalt in die Mordaktion führte über Zwischenverlegungen.

37 Vgl. Der Spiegel Nr. 42 vom 10.10.1971, 74-78.

38 Vgl. die Rezension von Thomas A. Knapp, The American Historical Review 77, 1972, $1148 \mathrm{f}$. sowie ähnlich Keith Robbins, The Journal of Ecclesiastical History 24, 1973, 103f. Dass der weitere Gang der Forschung auch das Bild des Kirchenkampfs verändert hat, sei hier nur angedeutet: Neben den Konfliktlinien treten Mischungsverhältnisse und Gemengelagen von Dissens und Kooperation stärker hervor. Vgl. u.a. Hans Günter Hockerts/Friedrich Wilhelm Graf (Hrsg.), Distanz und Nähe zugleich? Die christlichen Kirchen im „Dritten Reich“. München 2017. 